\title{
Detection of Startle-Type Epileptic Seizures using Machine Learning Technique
}

\author{
Pushpa Balakrishnan $^{1} \quad$ S. Hemalatha ${ }^{1}$ Dinesh Nayak Shroff Keshav² \\ ${ }^{1}$ Department of Electronics and Instrumentation Engineering, BSA \\ Crescent Institute of Science and Technology, Chennai, Tamil \\ Nadu, India \\ ${ }^{2}$ Department of Neurology, Gleneagles Global Hospital, Chennai, \\ Tamil Nadu, India

\begin{abstract}
Address for correspondence Pushpa Balakrishnan, M. Tech, Department of Electronics and Instrumentation Engineering BSA Crescent Institute of Science and Technology, Chennai, Tamil Nadu, India (e-mail: pushpa_akm@crescent.education).
\end{abstract}

Int J Epilepsy 2018;5:92-98

\begin{abstract}
Background Epilepsy is a common neurological disorder characterized by seizures and can lead to life-threatening consequences. The electroencephalogram (EEG) is a diagnostic test used to analyze brain activity in various neurological conditions including epilepsy and interpreted by the clinician for appropriate diagnosis. However, the process of EEG analysis for diagnosis can be automated using machine learning algorithms (MLAs) to aid the clinician. The objective of the study was to test different algorithms that could be used for the detection of seizures.

Materials and Methods Video EEG (vEEG) was collected from subjects diagnosed to have episodes of seizures. The epilepsy dataset thus obtained was subjected to empirical mode decomposition (EMD) and the signal was decomposed into intrinsic mode functions (IMFs). The first five levels of decomposition were considered for analysis as per the established protocol. Statistical features such as interquartile range (IQR), entropy, and mean absolute deviation (MAD) were extracted from these IMFs.

Results In this study, different MLAs such as nearest neighbor (NN), naïve Bayes (NB), and support vector machines (SVMs) were used to distinguish between normal

Keywords

- empirical mode decomposition

- seizures

- machine learning (interictal) and abnormal (ictal) states. The demonstrated accuracy rates were $97.32 \%$ for NN, $99.02 \%$ for NB, and $93.75 \%$ for SVM.

Conclusion Based on this accuracy and sensitivity, it may be posited that the NB classifier provides significantly better results for the detection of abnormal signals indicating that MLA can detect the seizure with better accuracy.
\end{abstract}

\section{Introduction}

Epilepsy is a common neurological disorder characterized by seizure activity in the brain. Globally, over 65 million people suffer from epilepsy. The most commonly occurring seizures $(60 \%)$ are convulsive in nature; the remaining $40 \%$ are nonconvulsive. ${ }^{1-3}$ Startle-type epilepsy as a form of reflex seizure in which startle activates the epileptic form discharge when a patient hears loud noises or sudden surprises. This is seen as an unconscious defensive response to sudden and threatening stimulus and is associated with a negative effect. It is a startle reflex reaction which serves as a mechanism to save vital parts. The subjects are specifically sensitive to

received

February 26, 2019

accepted after revision

May 21, 2019
DOI https://doi.org/

$10.1055 / \mathrm{s}-0039-1693072$

ISSN 2213-6320. one sensory modality such as temperature, taste, sound, and pressure. Sudden noise is one of the important triggers for provoked seizure. $^{4,5}$

Scalp electroencephalogram (EEG) is a primary test to diagnosis epilepsy. Gibbs and Gibbs (1951) described the original EEG patterns. Scalp EEG signals are nonlinear and nonstationary in nature. To analyze the signal, there are descriptive and heuristics methods. Most of the methods are based on domains such as time, frequency, time-frequency, and nonlinear methods. ${ }^{6,7}$ The EEG is a time series data that has nonlinear and nonstationary characteristics. Empirical mode decomposition (EMD) algorithm is applied to the EEG signals to extract intrinsic mode functions (IMFs). The mathematical

Copyright @2018 Indian Epilepsy Society

License terms

$($ (1) $\odot \circledast$ 
rationale is developed by Huang. ${ }^{8}$ IMFs provide instantaneous frequency as functions of time that furnish good identification of embedded structures. The IMF is based on local properties of the scalp EEG signal which makes the instantaneous frequency useful for analyzing the neuronal activity. This removes the need for spurious harmonics in the nonlinear and nonstationary signals. In classical method, the Fourier transform is used. There is a need for restrictive assumptions and conditions. ${ }^{9}$ Some of the variations by the way of smoothing and transforming are based on a priori assumptions. However, in the case of empirical mode decomposition, the output is amenable to statistical methods over a time domain without any restrictive assumptions. These data are suitable for a composite data response involving many variables.

The scalp EEG signal is determined based on the classification and the performance using machine learning algorithms (MLAs). ${ }^{7,10}$ Nonlinear parameters such as interquartile range (IQR) are used to characterize the scalp EEG signal into normal and abnormal. ${ }^{11}$ Feature extraction is performed based on IMF output. These features are mean absolute deviation (MAD), IQR, and entropy, and three different algorithms are used for classification. ${ }^{12,13}$

The objective of this study was to classify the scalp EEG signal using MLA, with better accuracy for startle-type epilepsy. In this study, the time-frequency domain is adapted. EMD algorithm is applied to the scalp EEG signals to extract IMFs.

\section{Materials and Methods}

\section{Study Site}

The research was conducted at Global Healthy City, Chennai, under the supervision of an epileptologist. The Neuro laboratory is equipped with digital monitoring video-EEG system (Nicolet One Neuro Diagnostic System; NATUS Neuro, Department of Neurology) commonly known as "epilepsy monitoring unit." The vEEG data were finally digitized, and the sampling rate of $256 \mathrm{~Hz}$ was recorded and stored. The methodology of the study is shown in - Fig. 1 .

\section{Study Subjects}

The subjects were individuals with epilepsy and recruited into the study through their parents as they are adolescents. The scalp vEEG data were acquired from 10 subjects with startle-type epilepsy (4 males and 6 females; age range 13-16 years). International standard $10-20$ system electrode placement was used, and a bipolar electrode montage was used for the diagnosis of the patient. Each electrode output is bandpass filtered by 0.5 to $100 \mathrm{~Hz}$ during recording by setting the low cut and high cut at $0.3 \mathrm{~Hz}$ and $70 \mathrm{~Hz}$, respectively.

\section{Preprocessing}

To remove artifacts in the signal and power line disturbance, the signal is preprocessed with a notch filter with a frequency of $50 \mathrm{~Hz} .{ }^{14}$

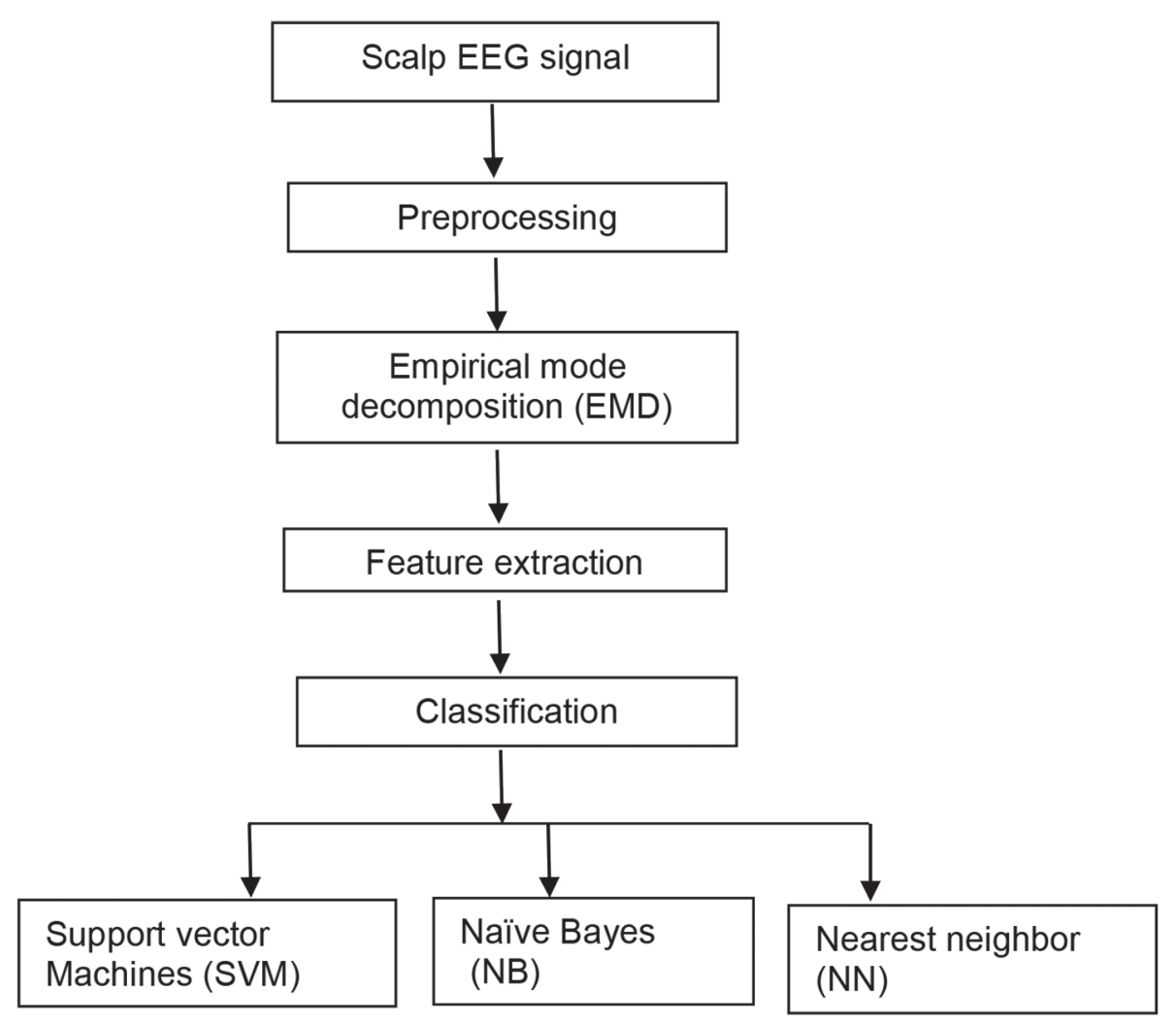

Fig. 1 Study methodology. 


\section{Empirical Mode Decomposition}

EMD is applied to the signals and it partitions the signal to elemental IMFs. These IMFs satisfy the following conditions:

1. Given an input signal $[y(t)]$, the number of zero crossings and extremes must be same or must vary by one.

2. At any instance, the mean value of the envelope illustrates the local minima. The local maxima must be zero. ${ }^{15,16}$

The algorithm used for the study is depicted in the accompanying flow chart (-Fig. 2).

\section{Feature Extraction}

The IMF coefficients are extracted to provide information that shows the distribution of the signal in time and frequency. The coefficients corresponding to the frequency bands
IMF1-IMF5 were analyzed, thus reducing the number of features that represent the signal. Statistical parameters such as entropy, IQR, and MAD of the signal were calculated for the frequency bands IMF1-IMF $5 .{ }^{17}$

\section{Entropy}

Entropy is a measure for quantifying the degree of complexity, irregularity of time series, and is used for extracting the information from the signal. It characterizes quantification of the complexity of brain dynamics. The signal uncertainty is measured in terms of the repeatability of the signal amplitude. ${ }^{18,19}$

$$
E(p)=-\sum_{i=1}^{N} p \log (p)
$$

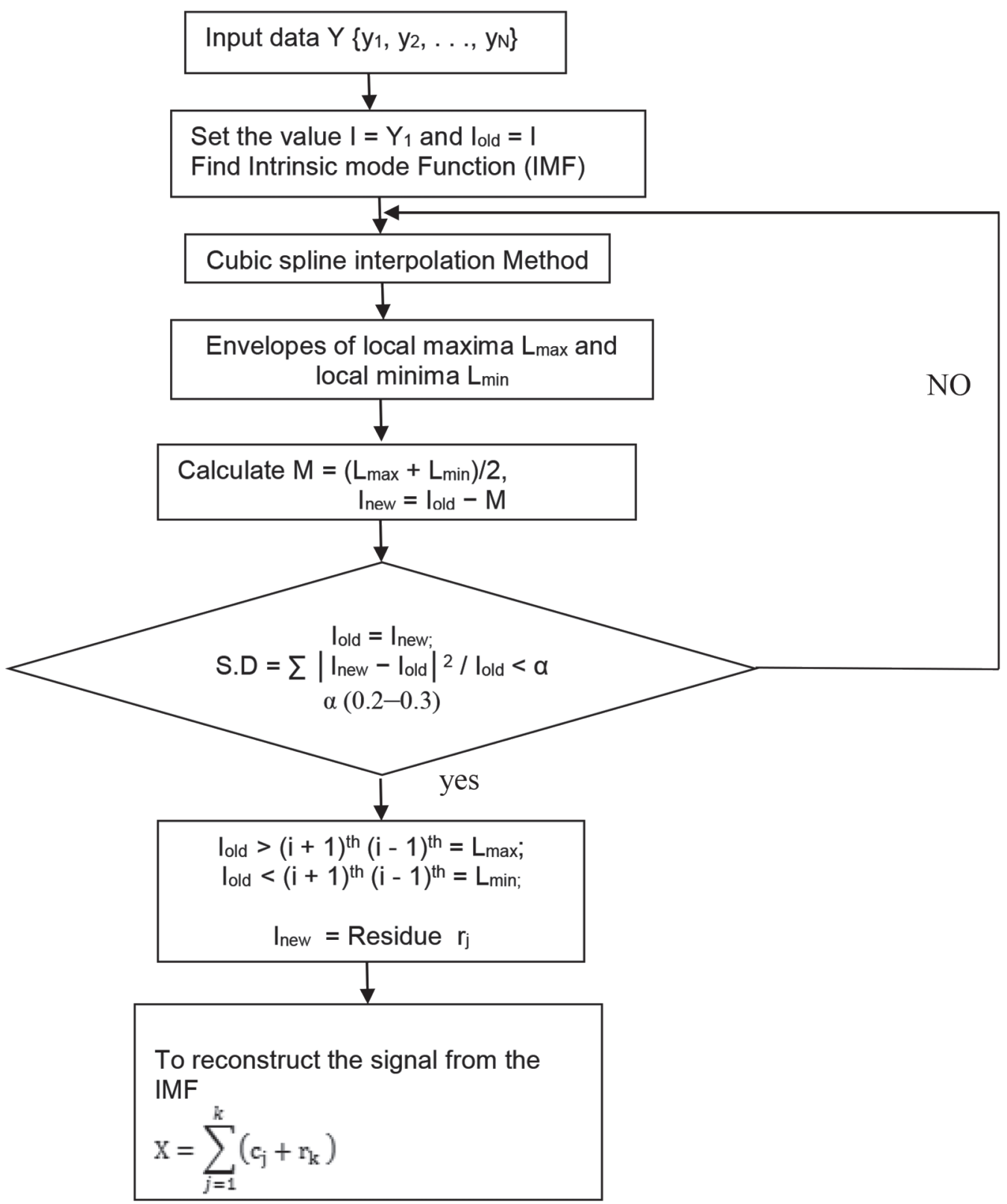

Fig. 2 Flowchart of empirical mode decomposition. 


\section{Interquartile Range}

The IQR is a measure of changeability and depends on dividing data into quartiles. Quartiles separate the ordered data into four equal parts. The values that divide each part are termed as the first, second, and third quartiles, denoted by , , and , respectively. ${ }^{20,21}$

\section{Mean Absolute Deviation}

The mean absolute deviation (MAD) measures the robustness of the collected quantitative data. The MAD of a dataset is the average distance between each data point and the mean. ${ }^{21}$

\section{Classification}

The research envisages finding the effectiveness of popularly used parametric and nonparametric algorithms to discriminate between normal and abnormal states within a patient and these algorithms are used to classify scalp vEEG signals. A parametric method, that is, support vector machine (SVM), and nonparametric methods, that is, naïve Bayes (NB) and nearest neighbor (NN), are used.22 The classification is performed using MATLAB functions with linear kernel, while all other parameters are set to default for increased accuracy. Features such as IQR, MAD, and entropy are applied to the classifier SVM, NB, and NN. ${ }^{23}$ The SVM is a well-known supervised learning algorithm for analyzing the data. It is based on the assumption of decision planes that separate a set of objects having different class memberships. For this type the training involves the minimization of error function.
This is subject to the following constraints:

$$
\frac{1}{2} w^{T} w+C \sum_{i=1}^{N} \xi i
$$

In above equations, $w$ is the vector of coefficients, $b$ is a

$$
y_{i\left(w^{t} \phi\left(x_{i}\right)+b\right) \geq 1-\xi i \text { and } \xi i \geq 0, i=1, \ldots . N}
$$

constant, and represents parameters for handling nonseparable data inputs. The NB classifier works on the probability theory, where model parameters are estimated according to the distribution of the training data. It computes the probability of the new object, with respect to each class, and then chooses the most probable class. The configuration of the NB function with Gaussian distribution is used. ${ }^{24} \mathrm{NN}$ is a nonparametric method that searches the $k$ value for optimality; $k=3$ increases the accuracy of the classification. ${ }^{24}$

\section{Ethics Approval}

Institutional Ethical Committee (IEC) approval was obtained before the start of the study. All subjects were explained the study details in their native language and recruited into the study after informed written consent. In the case of subjects younger than 18 years of age, informed written consent was obtained from the parents/guardian.

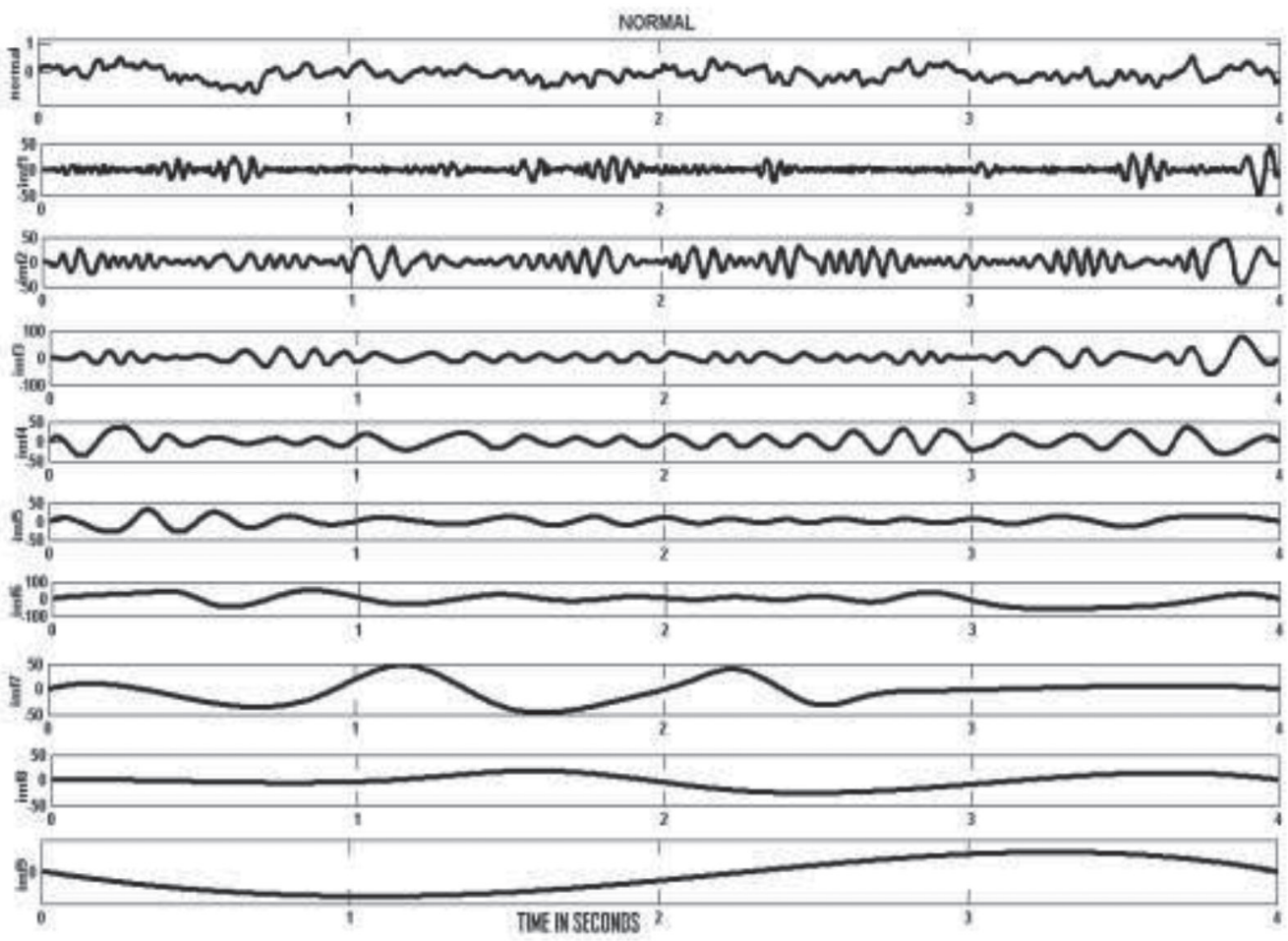

Fig. 3 Intrinsic mode function (IMF) of normal signal. 


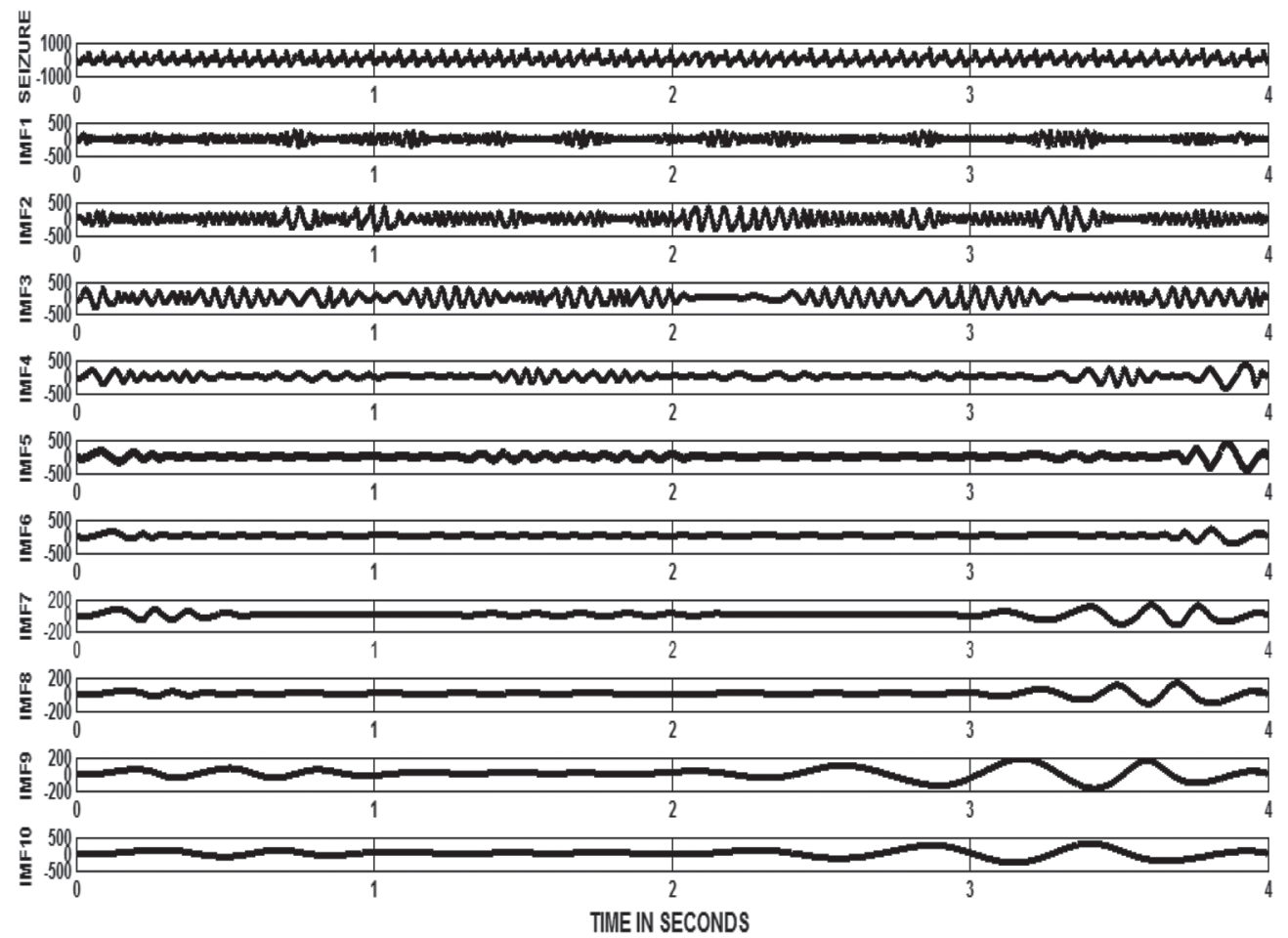

Fig. 4 Intrinsic mode function (IMF) of abnormal signal.

Table 1 Normal and abnormal features extracted from the IMF

\begin{tabular}{|l|l|l|l|l|l|}
\hline Normal features & IMF1 & IMF2 & IMF3 & IMF4 & IMF5 \\
\hline IQR & 2.0425 & 1.2506 & 1.5318 & 1.1900 & 1.9500 \\
\hline MAD & 1.9956 & 1.6489 & 1.9366 & 0.9954 & 1.3077 \\
\hline Entropy & 0.5456 & 0.5218 & 0.6548 & 0.4880 & 0.6293 \\
\hline Abnormal features & IMF1 & IMF2 & IMF3 & IMF4 & IMF5 \\
\hline IQR & 4.3740 & 4.5247 & 4.5331 & 4.2849 & 4.1366 \\
\hline MAD & 11.6847 & 15.4772 & 13.0137 & 12.1433 & 11.7174 \\
\hline Entropy & 1.9423 & 1.8483 & 1.5172 & 1.6890 & 1.7856 \\
\hline
\end{tabular}

Abbreviations: IMF, intrinsic mode function; IQR, interquartile range; MAD, mean absolute deviation.

\section{Results}

From the scalp vEEG signals, it is possible to differentiate between a normal to that of an abnormal signal. The subjects experienced normal as well as abnormal states. The corresponding IMF levels (up to 10) are depicted in - Figs. 3 and 4. The features extracted for the IMF are presented in - Table $\mathbf{1}$. From the data it may be posited that the features are clearly different for normal and abnormal EEG signals. Furthermore, these features were provided as inputs to classifiers during the training process. These extracted features were given to classifiers. From these data, we considered $70 \%$ for the training set, $20 \%$ for testing set, and $10 \%$ for validation. The performances of the algorithm are tabulated and shown in - Table 2. The discriminative potentials of the algorithms were quantified by diagnostic measures of accuracy given by sensitivity, specificity, positive prediction value, and negative prediction value.

\section{Discussion}

The analysis was undertaken to find the ability of different classifiers to classify data under supervised learning. Two parametric and one parametric algorithm were chosen. The data were subjected to analysis and the output of different algorithms are given in - Table 2. The comparison indicates that NB provides better indices compared with other methods and thus may be considered for further analysis. Higher values of sensitivity and specificity indicate that there is good discrimination between normal and abnormal signals of the subjects. The higher indices of NB algorithm may be attributed to the nonparametric nature of the algorithm. However, the results may not indicate NB as the test of choice for classification as the sample size was small. Furthermore, false-positive (FP) value is not possible as all the patients were diagnosed to have seizures, and epileptologist's diagnosis revealed that the subjects had startle-type epilepsy, and 
Table 2 Performance of the various indices corresponding to SVM, NB, and NN

\begin{tabular}{|l|l|l|l|}
\hline Indices & SVM (\%) & NB (\%) & NN (\%) \\
\hline Accuracy & 93.75 & 99.02 & 97.32 \\
\hline Sensitivity & 99.3 & 99.9 & 98.6 \\
\hline Specificity & 87.5 & 96 & 94 \\
\hline Positive predictive value & 88.89 & 94.25 & 95.26 \\
\hline Negative predictive value & 99.6 & 98.25 & 99.3 \\
\hline
\end{tabular}

Abbreviations: SVM, support vector machines; NB, naïve Bayes; NN, nearest neighbor.

it is one of the limitations of the study. Some subjects may be classified as not having a disease which is called falsenegative. Sensitivity is defined as a ratio of $(\mathrm{TP} /[\mathrm{TP}+\mathrm{FN}])$. Since the sensitivity is $99.9 \%$, indicating false-negative is of very low value. Positive predictive value is defined as the probability of having a state of interest in a subject with a positive result. The high value of positive predictive value indicates the discrimination between patients having the disease and not having the disease is very high. Among those where subject's data were analyzed, the positive predictive value was 94.25. The high value indicates that the algorithm is able to discriminate the abnormal and normal signals. The present study is an explanatory study using data of 10 adolescent subjects. The subjects had episodes of seizures and were on antiepileptic drugs. The small dataset may not be able to correlate the clinical profile with the results. Entropy measured in the present study is likely to be affected with mode misalignments and mode mixing problems for complex data such as brain signals. Diagnostic measures such as likelihood ratio, ROC curve, and Youden index are not considered for this study.

The present study is an explanatory study using data of 10 adolescent subjects. The subjects had episodes of seizures and are on antiepileptic drugs. The small dataset may not be able to correlate clinical profile with the results thus obtained. Entropy measured in the present study is likely to be affected with mode misalignments and mode mixing problems for a complex data such as brain signals. Diagnostic measures such as likelihood ratio, ROC curve, and Youden index are not included and are planned for future studies.

\section{Conclusion}

Using EMD, we have attempted to extract EEG features to help us distinguish between normal and seizure signals, by calculating MAD, IQR, and entropy. Accordingly, the EEG data are classified into normal and abnormal, using a machine learning technique. The performances of the algorithms are then compared. From this, it is observed that NB has slightly higher accuracy than both SVM and NN. Further studies seem necessary to enhance the detection accuracy and resolution; this could be made possible by including more features and by applying the Random Forest classifier for optimal results.

\section{Conflict of Interest}

None declared.

\section{References}

1 Capovilla G, Kaufman KR, Perucca E, Moshé SL, Arida RM. Epilepsy, seizures, physical exercise, and sports: a report from the ILAE Task Force on Sports and Epilepsy. Epilepsia 2016;57(1):6-12

2 Fisher RS, van Emde Boas W, Blume W, et al. Epileptic seizures and epilepsy: definitions proposed by the International League Against Epilepsy (ILAE) and the International Bureau for Epilepsy (IBE) Epilepsia 2005;46(4):470-472

3 Radhakrishnan A. Bridging the treatment gap in epilepsyis there an emerging trend in the use of newer antiepileptic drugs? Neurol India 2016;64(6):1140-1142

4 Andermann F, Keene DL, Andermann E, Quesney LF. Startle disease or hyperekplexia: further delineation of the syndrome. Brain 1980;103(4):985-997

5 Startle epilepsy (startle-induced epilepsy). Epilepsy Foundation Website. Available at: https://www.epilepsy. com/learn/professionals/about-epilepsy-seizures / reflex-seizures-and-related-epileptic-syndromes/startle

6 Sanariya K, Garg A, More A, Bansal AR. 6 and $14 \mathrm{~Hz}$ positive spikes on scalp electroencephalogram. Int J Epilepsy 2018 5(1):9-12

7 Acharya UR, Sree SV, Swapna G, Martis RJ, Suri JS. Automated EEG analysis of epilepsy: a review. Knowledge Base Systems 2013;45:147-165

8 Huang NE, Shen Z, Long SR, et al. The empirical mode decomposition and the Hilbert spectrum for nonlinear and non-stationary time series analysis. Proc R Soc Lond A1998;454(1971):903-995

9 Titchmarsh EC. Introduction to the theory of Fourier integrals. Oxford, UK: Oxford University Press 1948

10 Pushpa B, Najumnissa J, Keshav DN. Correlation analysis and modeling of EEG-EMG signal for startle-induced seizures. OnLine Journal of Biological Sciences 2017;2018, 18, (1):17-23

11 Kannathal N, Acharya UR, Lim CM, Sadasivan PK. Characterization of EEG-a comparative study. Comput Methods Programs Biomed 2005;80(1):17-23

12 Das AB, Mohammed IHB. Discrimination and classification of focal and non-focal EEG signals using entropy-based features in the EMD-DWT domain. Biomed Signal Process Control 2016;29:11-21

13 Martis RJ, Acharya UR, Tan JH, et al. Application of empirical mode decomposition (EMD) for automated detection of epilepsy using EEG signals. Int J Neural Syst 2012;22(6):1250027

14 Flandrin P, Rilling G, Goncalves P. Empirical mode decomposition as a filter bank. IEEE Signal Process Lett 2004; 11(2):112-114

15 Zhang Z, Parhi KK. Low-complexity seizure prediction from iEEG/sEEG using spectral power and ratios of spectral power. IEEE Trans Biomed Circuits Syst 2016;10(3):693-706

16 Lewandowski A, Williams DF, Hale P, Wang CMJ, Dienstfrey A. Covariance-based vector-network-analyzer uncertainty analysis for time-and frequency-domain measurements. IEEE Trans Microw Theory Tech 2010;58(7):1877-1886 
17 Tafreshi AK, Ali MN, Omidvarnia AH. Epileptic seizure detection using empirical mode decomposition. Presented at: IEEE international symposium on signal processing and information technology Sarajevo, Serbia; December 16-18; 2008

18 Pincus SM. Approximate entropy as a measure of system complexity. Proc Natl Acad Sci U S A 1991;88(6):2297-2301

19 Richman JS, Moorman JR. Physiological time-series analysis using approximate entropy and sample entropy. Am J Physiol Heart Circ Physiol 2000;278(6):H2039-H2049

20 Zhou W, Liu Y, Yuan Q, Li X. Epileptic seizure detection using lacunarity and Bayesian linear discriminant analysis in intracranial EEG. IEEE Trans Biomed Eng 2013;60(12):3375-3381

21 Ahammad N, Fathima T, Joseph P. Detection of epileptic seizure event and onset using EEG. BioMed Res Int 2014. doi: http:// dx.doi.org/10.1155/2014/450573
22 Guarnizo C, Delgado E, EEG single-channel seizure recognition using empirical mode decomposition and normalized mutual information. In: proceedings of IEEE 10th International Conference on Signal Processing (ICSP), Beijing, October 24-28, 20102010

23 Bernardo JM, Smith AFM. Bayesian Theory. 1st ed. Wiley; 2000

24 Parvinnia E, et al. Classification of EEG signals using adaptive weighted distance nearest neighbor algorithm. Journal of King Saud University-Computer and Information Sciences 2014;26(1):1-6 